Chirurg 2019 90 (Suppl 2):S18 https://doi.org/10.1007/s00104-019-0890-8 Online publiziert: 13. Februar 2019

(c) Springer Medizin Verlag GmbH, ein Teil von Springer Nature 2019

\section{Originalpublikation}

Parise P, Ferrari C, Cossu A et al (2018) Enhanced recovery after surgery (ERAS) pathway in Esophagectomy. Is a reasonable prediction of hospital stay possible? Ann Surg https://doi.org/10.1097/SLA000002775. [Epub ahead of print]

Hintergrund und Fragestellung. „Enhanced-recovery-after-surgery"(ERAS)Protokolle (Synonym: Fast-Track-Protokolle) haben sich in der kolorektalen Chirurgie als fester Bestandteil des perioperativen Managements etabliert und nachweislich die perioperative Morbidität reduziert. Die Implementierung von ERAS-Protokollen in der Ösophaguschirurgie ist deutlich protrahierter und die gegenwärtige Studienlage zeigt widersprüchliche Ergebnisse hinsichtlich einer Reduktion der postoperativen Morbidität. Insbesondere die stringente und vollständige Umsetzung von ERASProtokollen nach Ösophagektomie ist schwierig. Zu diesem Thema wurde von einer italienischen Arbeitsgruppe eine Studie mit dem Ziel initiiert, ein ScoringSystem zu entwickeln, welches anhand definierter Variablen die inkomplette Umsetzung eines ERAS-Protokolls und damit verzögerte Entlassung vorhersagen kann.

Methoden. In diese prospektive Beobachtungsstudie wurden insgesamt 286 Patienten mit Karzinom des Ösophagus oder des gastroösophagealen

Dieser Beitrag wurde erstpubliziert in Der Chirurg (2018) 89:642-642. https://doi.org/10. 1007/s00104-018-0649-7

W. Schröder · C. J. Bruns

Klinik für Allgemein-, Viszeral- und Tumorchirurgie, Universitätsklinik Köln, Köln, Deutschland

\title{
Fast-Track-Protokolle in der Ösophaguschirurgie - was ist machbar?
}

Übergangs eingeschlossen (Zeitraum 04/2012-03/2014). 86,3\% der Patienten wurden transthorakalösophagektomiert, $13,0 \%$ transhiatal erweitert gastrektomiert. Bei 25,5\% der Patienten wurde der Eingriff primär offen durchgeführt, bei $30,5 \%$ total minimal-invasiv und bei 39,5\% als Hybridverfahren (Laparoskopie und Thorakotomie). Alle Patienten wurden entsprechend einem standardisierten perioperativen ERAS-Protokoll geführt, die Entlassung aus der stationären Behandlung war für den 8 . postoperativen Tag geplant. 22 Variablen mit möglichem Einfluss auf den postoperativen Verlauf wurden analysiert und ein Score zur Vorhersage des Zielparameters „Entlassung $\leq 8$. postoperativer Tag“ berechnet.

Ergebnisse. In der multivariaten Regressionsanalyse war ein ASA-Score $\geq 3$, eine Operationsdauer $>255 \mathrm{~min}$, eine „Nichthybrid“-Ösophagektomie und eine protrahierte Mobilisation des Patienten $>24 \mathrm{~h}$ mit einer statistisch signifikant verzögerten Entlassung nach dem 8. postoperativen Tag assoziiert. Das Regressionsmodel bezogen auf die Entlassung nach dem 8. postoperativen Tag hatte einen positiven Vorhersagewert von $81,9 \%$, die Sensitivität betrug $96,6 \%$ und die Spezifität 17,6\%. Der aus diesen vier Variablen berechnete Enhanced Recovery Predicting Score wurde bei 23 Patienten prospektiv untersucht und konnte bei allen Patienten den Entlassungstag ( $\leq$ vs. $>8$. postoperativer Tag) korrekt identifizieren, bei 21 Patienten erfolgte die Entlassung nach dem 8. postoperativen Tag.
Fazit. Die Studie zeigt, wie schwierig die frühzeitige Entlassung nach Ösophagektomie auch in einem ERAS-Protokoll umzusetzen ist. Von Bedeutung ist aber, dass 3 von 4 Variablen (Mobilisation, Operationsverfahren und -dauer) durch Anpassungen des peri- und intraoperativen Managements $\mathrm{zu}$ beeinflussen sind und damit die Liegedauer zumindest bei gesunden Pateinten (ASA 1 und 2) verkürzt werden kann, dass aber alle weiteren Variablen eines ERASProtokolls mit einem standardisierten „clinical pathway“ keinen Einfluss auf die Liegedauer haben. Insbesondere die frühzeitige und intensive Mobilisation nach Ösophagektomie ist aber von entsprechenden Personalressourcen abhängig, um das Ziel einer frühzeitigen Entlassung $\mathrm{zu}$ erreichen. Wichtig erscheint auch, dass alle Patienten dieser Studie frühzeitig über eine Katheterjejunostomie enteral ernährt wurden. In diesen beiden Aspekten, Ernährung und Mobilisation, ist eine Anpassung des postoperativen Managements für alle Patienten nach Ösophagektomie zu diskutieren.

\section{Korrespondenzadresse}

Prof. Dr. W. Schröder, FACS, FEBS

Klinik für Allgemein-, Viszeral- und Tumorchirurgie, Universitätsklinik Köln Kerpener Str. 62, 50937 Köln, Deutschland wolfgang.schroeder@uni-koeln.de

Interessenkonflikt. W.Schröder und C.J. Bruns geben an, dass kein Interessenkonflikt besteht. 